\begin{tabular}{|l|l|l|l|l|l|l|l|l|l|l|l|l|l|l|}
\hline 1 & 1 & 1 & 1 & 1 & & 0 & 0 & 0 & 1 & 1 & 1 & 1 & 1 & 1 \\
\hline 1 & 1 & 1 & 1 & 1 & 1 & 0 & & 0 & 1 & 1 & 1 & 1 & 1 & 1 \\
\hline 1 & 1 & 1 & 1 & 1 & 1 & 0 & 0 & 1 & 1 & 1 & 1 & 1 & 1 \\
\hline 1 & 1 & 1 & 1 & 1 & 0 & 0 & & 0 & 0 & & 1 & 1 & 1 & 1 \\
\hline 1 & 1 & 1 & 1 & 1 & 0 & 0 & & 0 & 0 & 1 & 1 & 1 & 1 & 1 \\
\hline 1 & 1 & 1 & & 0 & 0 & 0 & & 0 & 0 & 0 & 1 & 1 & 1 & 1 \\
\hline 1 & 1 & 1 & 1 & 0 & 0 & 0 & & 0 & 0 & 0 & 1 & 1 & 1 & 1 \\
\hline 1 & 1 & 1 & 0 & 0 & 0 & 0 & & 0 & 0 & 0 & & 1 & 1 \\
\hline 1 & 1 & 1 & 1 & 0 & 0 & 0 & 0 & 0 & 0 & 0 & 1 & 1 & 1 & 1 \\
\hline 1 & 1 & 1 & 1 & 0 & 0 & 0 & & & 0 & 7 & 1 & 1 & 1 \\
\hline 1 & 1 & 1 & 1 & 1 & & & & & 1 & 1 & 1 & 1 & 1 \\
\hline 1 & 1 & 1 & 1 & 1 & 0 & 0 & 0 & & & 1 & 1 & 1 & 1 \\
\hline 1 & 1 & 1 & 1 & 1 & 1 & 0 & 0 & 0 & 1 & 1 & 1 & 1 & 1 & 1 \\
\hline 1 & 1 & 1 & 1 & 1 & 1 & 0 & 0 & 0 & 1 & 1 & 1 & 1 & 1 & 1 \\
\hline 1 & 1 & 1 & 1 & 1 & 1 & 0 & 0 & 0 & 1 & 1 & 1 & 1 & 1 & 1 \\
\hline
\end{tabular}




\section{Towards Accurate Porosity Descriptors based on Guest-Host Interactions}

Dooam Paik ${ }^{\mathrm{a}, \mathrm{d}}$, Maciej Haranczyk ${ }^{\mathrm{b}, \mathrm{c}, \mathrm{e}}$, and Jihan Kim ${ }^{\mathrm{a}, \mathrm{f}}$

${ }^{a}$ Department of Chemical and Biomolecular Engineering, KAIST, 291 Daehak-ro,

Yuseong-gu, Daejeon, Republic of Korea

${ }^{b}$ Lawrence Berkeley National Laboratory, Berkeley, California, 94720, United States

${ }^{\mathrm{c}}$ IMDEA Materials Institute, Calle Eric Kandel, 2, 28906 Getafe, Madrid, Spain

d libimor@kaist.ac.kr

e mharanczyk@lbl.gov

f jihankim@kaist.ac.kr 
ABSTRACT: For nanoporous materials at the characterization level, geometry-based approaches have become the methods of choice to provide information, often encoded in numerical descriptors, about the pores and the channels of a porous material. Examples of most common descriptors of the latter are pore limiting diameters, accessible surface area and accessible volume. The geometry-based methods exploit hard-sphere approximation for atoms, which (1) reduces costly computations of the interatomic interactions between the probe guest molecule and the porous material framework atoms, (2) effectively exploit applied mathematics methods such as Voronoi decomposition to represent and characterize porosity. In this work, we revisit and quantify the shortcoming of the geometry-based approaches. To do so, we have developed a series of algorithms to calculate pore descriptors such as void fraction, accessible surface area, pore limiting diameters (largest included sphere, and largest free sphere) based on a classical force field model of interactions between the guest and the framework atoms. Our resulting energy-based methods are tested on diverse sets of metal-organic frameworks and zeolite structures and comparisons against results obtained from geometric-based method indicate deviations in the cases for structures with small pore sizes. The method provides both high accuracy and performance making it suitable when screening a large database of materials.

KEYWORDS Porous Material, Void Fraction, Surface Area, Largest Included Sphere, Largest Free Sphere 


\section{Introduction}

Nanoporous materials contain intrinsic void spaces, which can be penetrated by guest molecules and exploited in a variety of applications including carbon capture, gas storage, catalysis, and drug delivery [1-4]. Nanoporous materials include several families of materials such as zeolites, metal organic frameworks (MOFs), covalent organic frameworks (COFs), porous polymer networks (PPNs) and related families of materials, which contain virtually unlimited number of possible structures. MOFs, for example, consist of metal atoms and organic linkers that can be linked together in variety of different ways due to a wide selection of possible metals and ligands as well as their compatible topologies. Accordingly, the number of experimentally synthesized MOFs has exceeded 5000 [5], while even greater number of predicted MOF structures in hypothetical MOF database awaits experimental confirmation [6]. Similarly, databases of predicted and synthesized zeolites, COFs and other porous structures have been collected and made available to the community [7-9].

The current state-of-the-art methodologies based on molecular simulations and/or electronic structure calculations allow for prediction of guest-related adsorption and diffusion properties of a porous material [10]. The undisputed workhorse underlying such predictions are classical force fields, which provide models (and parameters) describing the interaction potential between atoms of the material framework and the guest molecules. With proper parameterization based on fitting to either experimental or $a b$ initio-predicted data, material properties such as Henry's constants, adsorption isotherms, and diffusion coefficients can be reliably predicted [11-13]. The molecular simulations can also be used 
in high-throughput manner, exploiting parallel computing architectures, to predict properties and screen very large sets of structures. For example, Wilmer et al. have conducted methane storage screening on over 100000 hypothetical MOF structures to identify structures with high methane uptake properties [6]. Lin et al. have developed a metric of parasitic energy and screened a large database of zeolites and ZIF structures to identify materials best for carbon capture [14]. Kim et al. have screened over 80000 pure silica zeolite structures for $\mathrm{CH}_{4} / \mathrm{CO}_{2}$ selective materials [15]. Haldoupis et al. have screened over 250000 porous materials for various diffusion properties [16]. More recently, Simon et al. have screened the most of the available large material datasets (500k+ structures), referred-to as the Nanoporous Materials Genome, in the context of both methane storage and noble gas separations [17-18].

In typical studies of porous materials, oriented towards either small, e.g. one-to-few, or large sets of materials, there is a need to characterize particular pores or entire porosity of a structure. In the past, there have been many research reports on methods as well as software codes to compute the porosity characteristics [19-22]. These are typically simplified to a number of numerical descriptors such as void fraction, accessible surface area, pore limiting diameters or vectors like pore size distributions. Vast majority of reported methods consider solely the geometry of the framework atoms and the spherical probe. These geometry-based methods assume that (1) atoms are hard spheres of certain, chemistry-determined radii and (2) the porosity corresponds with a region that can be occupied by the probe center without overlapping with any of the framework atoms. Following this definition, porosity descriptors like largest diameter of included sphere and 
free sphere (Di and Df, respectively), void fraction (VF) and accessible surface area (ASA) have been introduced [19]. Di and Df indicates the largest diameter of the probe that can respectively, occupy or diffuse through the pores. VF corresponds to the volume that can be occupied by the center of a probe with a given radius without overlapping with the framework's atoms. Similarly, ASA integrates the surface that defines the probe-accessible volume in the VF calculation.

The geometry-based methods allow reductions in costly computations of the interatomic interactions between the probe guest molecule and the porous material framework atoms, and effectively exploit applied mathematics methods such as Voronoi decomposition to represent and characterize porosity. Moreover, for certain applications like methane and hydrogen storage, these geometrical properties correlate well with material performances $[18,23]$ and compared to the more computational intensive Monte Carlo or molecular dynamics simulation codes, the geometric-based tools can be used to quickly screen through a very large database of porous materials and be embedded as a part of a scoring function in optimization-based design approaches [24, 25]. The geometrybased tools have become the methods of choice when (pre)screening large sets of materials mainly due to their non-prohibitive computational cost. However, the effect of their shortcomings and approximations that comes in particular from reducing any interactions to a binary, nearest neighbour functions, has not yet been systematically investigated.

In this work, we present a series of algorithms to calculate void fraction, surface area, largest included sphere, and the largest free sphere for a given pair of material and guest molecule. Our approach involves a force field model of guest-host interaction, and 
therefore we refer to it as energy-based approach. These contributions have allowed us to cross-examine the energy-based and the geometrical-based algorithms to provide a better understanding and quantify the errors coming from the shortcoming of the latter approach. The paper is organized as follows. In Section 2, the energy-based algorithm is explained in detail. In Section 3, simulation results obtained for a selected set of metal-organic framework and zeolite structures are shown for both energy-based and geometric-based methods. In Section 4, a concise summary of the work is presented with possible future work with regards to this topic.

\section{Methodology}

The scope of our energy-based method focuses on crystalline porous materials. Amorphous materials can be treated as well provided that an assumption about their periodicity on a (large) spatial scale is made. Given the repetitive nature of the crystalline materials, the energy-based method analysis is conducted on a unit cell of the porous material with imposed periodic boundary conditions. On top of the unit cell, a three-dimensional energy grid with uniform spacing ( $\Delta=0.15$ Angstroms) is constructed. The code was constructed such that the user can easily change the grid sizes along all three spatial directions. Each grid point represents the two-body potential energy between a probe molecule placed at the grid point and all of the framework atoms as well as their periodic images within 12.8 Angstroms of cutoff distance. In all of our work, methane and helium probes are used given their simplicity (e.g. these particles can be represented by a single point and long- 
range electrostatic interaction energies can be omitted) and the Lennard-Jones potential is used to model the potential energy interactions. For the host atom force field parameters, UFF is used for all of the metal organic framework (MOF) atoms [26] and Garcia-Perez force fields are used for zeolites [27]. For the methane probe, the Trappe force field is used as it has been shown to reproduce the vapor-liquid curve as well as predict accurately adsorption isotherm data in various porous materials [28]. Helium force field parameters are taken from Dubbeldam et al. [29].

The energy-based code was written from scratch using $\mathrm{C}++$ and is available upon request. For comparison purposes, we used Zeo++ code, which is a geometric-based code developed by Willems et al. [19], using high accuracy routines of Ref [30]. Four important properties of porous materials (void fraction, surface area, largest included sphere, and largest free sphere) are obtained using both of the methods as we present the details behind the energy-based method in what is to follow.

\subsection{Void fraction}

Void fraction measures the fraction of empty space found within the porous material. In general, the void fraction is inversely proportional to the framework density as the presence of framework atoms take up empty volume. In the two extreme cases, a void fraction of one indicates complete absence of framework atoms while a void fraction of zero indicates nonporous materials. In our energy-based methodology, the void fraction is determined from the potential energy values assigned to the energy grid points. After computing the energy grid, an additional binary grid (comprised of 0's and 1's) is constructed where an energy 
threshold value of $15 \mathrm{k}_{\mathrm{B}} \mathrm{T}$ is used to assign the binary grid values into either accessible (set to 0 ) or inaccessible (set to 1 ). $15 \mathrm{k}_{\mathrm{B}} \mathrm{T}$ was chosen as a reasonable criterion that determines accessibility in a typical experimental time-scale [31]. Upon obtaining the binary grid, a flood fill algorithm is conducted to identify regions of 0's that are surrounded by 1's in all spatial directions, thereby identifying and blocking inaccessible regions for a given probe The detail behind the flood fill algorithm is explained elsewhere [31]. Finally, the void fraction is computed from the binary grid as the ratio between the number of 0's and the total number of grid points (i.e. sum of 0's and 1's).

\subsection{Surface area}

The surface area of porous materials quantifies the amount of material exposed to and available to interact with the penetrating gas/liquid phase. For application purposes, the surface area may correlate well with, for example, gas storage capacity. To compute the surface area using an energy-based method, the previously defined binary grid is re-used. In order to determine the interface that separates the accessible/inaccessible regions, a subset of 0's (i.e. accessible grid points) that have at least one neighbouring 1's (i.e. inaccessible grid points) is identified. Here, a neighbour refers to any grid point that is separated by $\pm \Delta$ distance in any of the three spatial directions, where $\Delta=0.15$ Angstroms.

The surface area algorithm iterates through all the 0's, inspects the values of its 26 neighbour grid points and identifies all the 0's that are on the surface (where the number comes from a rectangular $3 \times 3 \times 3=27$ grid points with the center point having 26 neighbours). To aid in visualization, a two-dimensional binary grid that illustrates this 
concept is shown in Figure 1. A single grid point identified on the surface is converted to a

\begin{tabular}{|lllllllllll|}
\hline 1 & 1 & 1 & 1 & 1 & 1 & 1 & 1 & 1 & 1 & 1 \\
1 & 1 & 1 & 1 & 0 & 0 & 0 & 1 & 1 & 1 & 1 \\
1 & 1 & 1 & 0 & 0 & 0 & 0 & 0 & 1 & 1 & 1 \\
1 & 1 & 0 & 0 & 0 & 0 & 0 & 0 & 0 & 1 & 1 \\
1 & 0 & 0 & 0 & 0 & 0 & 0 & 0 & 0 & 0 & 1 \\
1 & 0 & 0 & 0 & 0 & 0 & 0 & 0 & 0 & 0 & 1 \\
1 & 0 & 0 & 0 & 0 & 0 & 0 & 0 & 0 & 0 & 1 \\
1 & 1 & 0 & 0 & 0 & 0 & 0 & 0 & 0 & 1 & 1 \\
1 & 1 & 1 & 0 & 0 & 0 & 0 & 0 & 1 & 1 & 1 \\
1 & 1 & 1 & 1 & 0 & 0 & 0 & 1 & 1 & 1 & 1 \\
1 & 1 & 1 & 1 & 1 & 1 & 1 & 1 & 1 & 1 & 1 \\
\hline
\end{tabular}

unit area of $\Delta^{2}$ Angstroms ${ }^{2}$ for selected materials, we have confirmed that as $\Delta$ decreases, the surface area converges to a set value. For normalization purposes, the total surface area can be divided by the total unit cell volume or mass, giving volumetric and gravimetric surface area, respectively.

Figure 1. Two-dimensional cross section of the porous material binary grid. ' 0 ' represents accessible and '1' represents inaccessible points. Red 0's are accessible points that lie on the surface area interface. Adding areas of the orange rectangle regions leads to the total surface area.

\subsection{Largest included sphere}

The largest included sphere quantifies the diameter of a maximum sphere that can fit inside the porous material. Similar to the void fraction and the surface area calculations, the binary grid is used to obtain the diameter of the largest included sphere. First, the method identifies all the 1 's (i.e. inaccessible grid points) that has at least a single 0 neighbouring 
grid point. To simplify notations, these points are labelled ' $1-0$ ' points and they effectively represent set of inaccessible points that are closest to the accessible regions. This procedure is conducted to reduce the number of interactions that need to be checked to identify the largest sphere. Next, distance between a given 0 grid point and all of the 1-0 grid points are computed. The minimum distance represents the radius of the largest sphere that can be fit inside the porous material while centered at this specific grid point. Finally, in order to find the largest included sphere, the algorithm iterates through all 0 grid points to obtain the maximum radius from all of the minimum distances. A two-dimensional illustration is shown in Figure 2. The total number of distance values that needs to be computed in this algorithm (product of all 0 grid points and all 1-0 grid points and their periodic images) can be over 100 million for a sufficiently large porous material, making the routine potentially very slow. Thus, to expedite this algorithm, the routine selectively samples the 0 through a three-stage process. In the first stage, only $1 / 64$ of the total 0 points are selected as minimum distance values are evaluated only for every $4^{\text {th }}$ grid point along $\mathrm{x}, \mathrm{y}$, and $\mathrm{z}$ directions. From the maximum distance identified from the first stage, every $2^{\text {nd }}$ grid point along the $\mathrm{x}, \mathrm{y}$, and $\mathrm{z}$ directions are selected with the total volume spanning $1 / 5$ of the accessible region centered at the initially identified maximum grid point. Finally, in the last stage, all points along $\mathrm{x}, \mathrm{y}$, and $\mathrm{z}$ directions are selected and evaluated in a volume spanning $1 / 2$ of the previous volume to identify the largest included sphere. The three-stage algorithm provides an order of magnitude speed up in the computational wall time while providing values that are within 5\% of the largest included sphere computed while iterating through all 0 points in a few selected MOF structures. The computational 
performance difference between the regular and the three-stage algorithms are included in the Supporting Information for a few selected structures. (See Supporting Information Table 1.)

\begin{tabular}{|lllllllllll|}
\hline 1 & 1 & 1 & 1 & 0 & 0 & 0 & 1 & 1 & 1 & 1 \\
1 & 1 & 1 & 1 & 0 & 0 & 0 & 1 & 1 & 1 & 1 \\
1 & 1 & 1 & 0 & 0 & 0 & 0 & 0 & 1 & 1 & 1 \\
1 & 1 & 1 & 0 & 0 & 0 & 0 & 0 & 1 & 1 & 1 \\
1 & 1 & 0 & 0 & 0 & 0 & 0 & 0 & 0 & 1 & 1 \\
1 & 1 & 0 & 0 & 0 & 0 & 0 & 0 & 0 & -1 & 1 \\
1 & 1 & 0 & 0 & 0 & 0 & 0 & 0 & 0 & 1 & 1 \\
1 & 1 & 1 & 0 & 0 & 0 & 0 & 0 & 1 & 1 & 1 \\
1 & 1 & 1 & 0 & 0 & 0 & 0 & 0 & -1 & 1 & 1 \\
1 & 1 & 1 & 1 & 0 & 0 & 0 & 1 & 1 & 1 & 1 \\
1 & 1 & 1 & 1 & 0 & 0 & 0 & 1 & 1 & 1 & 1 \\
\hline
\end{tabular}

Figure 2. Two-dimensional cross section of the porous material binary grid. The red 1's represent non-accessible points located on the surface of the porous material. The black lines between 0 's and 1's are the minimum distance for that 0 grid point (not all of them are shown for visual clarity). The maximum value of the radius (indicated by the red arrow) is obtained from a circle (in two-dimension) centered on the blue 0 grid point.

\subsection{Largest free sphere}

The largest free sphere refers to the maximum size of a sphere probe that can traverse across a single periodic translation in a crystalline material. This property is informative in 
determining the diffusion limitations imposed on the guest gas molecule inside a given material. In the energy-based methodology, the sphere is deemed to be free if it can traverse across a crystalline material from one side to another side along the same spatial direction without encountering a binary grid value of 1 in its path. In the algorithm, a probe of radius of $r_{0}$ is first assigned to test its traversal. If it succeeds in traversal, the radius is increased by $2 r_{0}$ and the algorithm restarts. If it fails, the radius is set to be the average of the failed probe size and the last successful probe size. Accordingly, the algorithm reduces the error of the maximum radius by half in each of the step leading to linear convergence.

For a given probe size of $r_{0}$, the algorithm begins by examining three sides of the unit cells (each of sides that face to $\mathrm{xy}, \mathrm{yz}$, and zx plane) to determine the possible entrance for the spherical probe. Within the two-dimensional surfaces along the sides, if there is a circle of binary grid points with all 0's that can fit $r_{0}$, then the path for traversal starts in this region (Figure 3(a)). The spherical probe moves along the forward direction away from the entrance $(+x$ for $y z$ plane entrance, $+y$ for $x z$ plane entrance, and $+z$ for $x y$ plane entrance) and the traversal is considered successful upon exiting through the other side of the unit cell corresponding to the same position as its starting point. If the probe encounters a dead end due to collection of 1 's, then the probe back tracks to its original position while blocking out the dead end regions into strings of 1 's such that the probe will not retrace back to this path. Accordingly, the probe can identify an alternative pathway (if there exists one) to continue on with the traversal. At the end, if the probe finds a path in any of the three directions, then it is deemed to be accessible. If the probe cannot find any more pathways during a given iteration, then the probe is deemed to be too large to diffuse through and the 
iteration ends followed by a new trial entrance (Figure 3(b)). If for all entrances, the probe fails to make it to the other side, then the probe size needs to be reduced according to the binary search algorithm.

\begin{tabular}{|lllllllllll|lllllllllll|}
\hline 1 & 1 & 1 & 1 & 1 & 1 & 1 & 1 & 1 & 1 & 1 & 1 & 1 & 1 & 1 & 1 & 1 & 1 & 1 & 1 & 1 & 1 \\
1 & 0 & 0 & 0 & 0 & 1 & 1 & 1 & 1 & 1 & 1 & 1 & 1 & 1 & 1 & 1 & 1 & 1 & 1 & 1 & 1 & 1 \\
0 & 0 & 0 & 0 & 1 & 1 & 1 & 1 & 1 & 1 & 1 & 1 & 1 & 1 & 1 & 1 & 1 & 1 & 1 & 1 & 1 & 1 \\
1 & 0 & 0 & 0 & 1 & 1 & 1 & 1 & 0 & 0 & 0 & 1 & 1 & 1 & 1 & 1 & 1 & 1 & 1 & 0 & 0 & 0 \\
1 & 0 & 0 & 1 & 1 & 1 & 1 & 0 & 0 & 0 & 0 & 1 & 1 & 1 & 1 & 1 & 1 & 1 & 0 & 0 & 0 & 0 \\
1 & 1 & 1 & 1 & 0 & 0 & 1 & 1 & 0 & 0 & 0 & 1 & 1 & 1 & 1 & 0 & 0 & 1 & 1 & 0 & 0 & 0 \\
1 & 1 & 1 & 1 & 0 & 0 & 0 & 1 & 1 & 0 & 0 & 1 & 1 & 1 & 1 & 0 & 0 & 0 & 1 & 1 & 0 & 0 \\
1 & 1 & 1 & 0 & 0 & 0 & 0 & 1 & 1 & 1 & 0 & 1 & 1 & 1 & 0 & 0 & 0 & 0 & 1 & 1 & 1 & 0 \\
1 & 1 & 1 & 1 & 0 & 0 & 0 & 1 & 1 & 1 & 1 & 1 & 1 & 1 & 1 & 0 & 0 & 0 & 1 & 1 & 1 & 1 \\
1 & 1 & 1 & 1 & 0 & 1 & 1 & 1 & 1 & 1 & 1 & 1 & 1 & 1 & 1 & 0 & 1 & 1 & 1 & 1 & 1 & 1 \\
1 & 1 & 1 & 1 & 1 & 1 & 1 & 1 & 1 & 1 & 1 & 1 & 1 & 1 & 1 & 1 & 1 & 1 & 1 & 1 & 1 & 1 \\
\hline
\end{tabular}

Figure 3. (a) A two-dimensional cross section of a unit cell in the binary grid space. The circle represents the acceptable entrance for the test probe with some given radius. (b) The newly selected position for the same probe upon failure to find a path from (a). The blue region represents the next candidate if the current one fails.

\section{Results}

To verify the accuracy of the energy-based method algorithms, 100 randomly selected MOFs were chosen from the CORE MOF database, which is a database of experimental MOF structures compiled by Chung et al. [32]. For each of the MOF structures, the void fraction (VF), surface area (SA), diameter of the largest included sphere (Di) and the diameter of the largest free sphere (Df) were obtained using both the energy-based method and the geometric method. 
A methane probe is used to obtain the four quantities (i.e. VF, SA, Di, and Df) for both the energy-based method (three different cutoff energy values: $5 \mathrm{k}_{\mathrm{B}} \mathrm{T}, 10 \mathrm{k}_{\mathrm{B}} \mathrm{T}$, and $\left.15 \mathrm{k}_{\mathrm{B}} \mathrm{T}\right)$ and geometric-based method as shown in Figure 4. In general, the two methods yield data points that are positively correlated, indicating good agreement. For larger energy cutoff, the proportion of 0's increase in the binary grid leading to increased VF, SA, Di, and Df. Overall, the energy-based method yields larger VF compared to the geometricbased method as the latter uses a hard-sphere model that underestimates adsorption. As such, there is a few number of porous materials for which methane is deemed to be nonporous using the geometric-based method but porous via the energy-based method. This reflects a softer nature of the Lennard-Jones potential in comparison to the hard sphere approximation of geometry-based approach. For SA, the discrepancy in SA computed between the energy-based and geometric-based method increases for larger SA. In general, it is conceivable that the energy-based method overestimates the surface area as all 0's that neighbours even a single inaccessible point are counted in the total SA. In order to account for this discrepancy, the curvature of the surface area might need to be taken into account, which can lower the SA in general for the energy-based method. For Di and Df, the energy-based method yields smaller values compared to the geometric-based method as the former obtains the maximum distance between an accessible point and the cutoff value (e.g. $\left.15 \mathrm{k}_{\mathrm{B}} \mathrm{T}\right)$ whereas the latter obtains the maximum distance between an accessible point and the framework atom (which has infinite potential energy). 

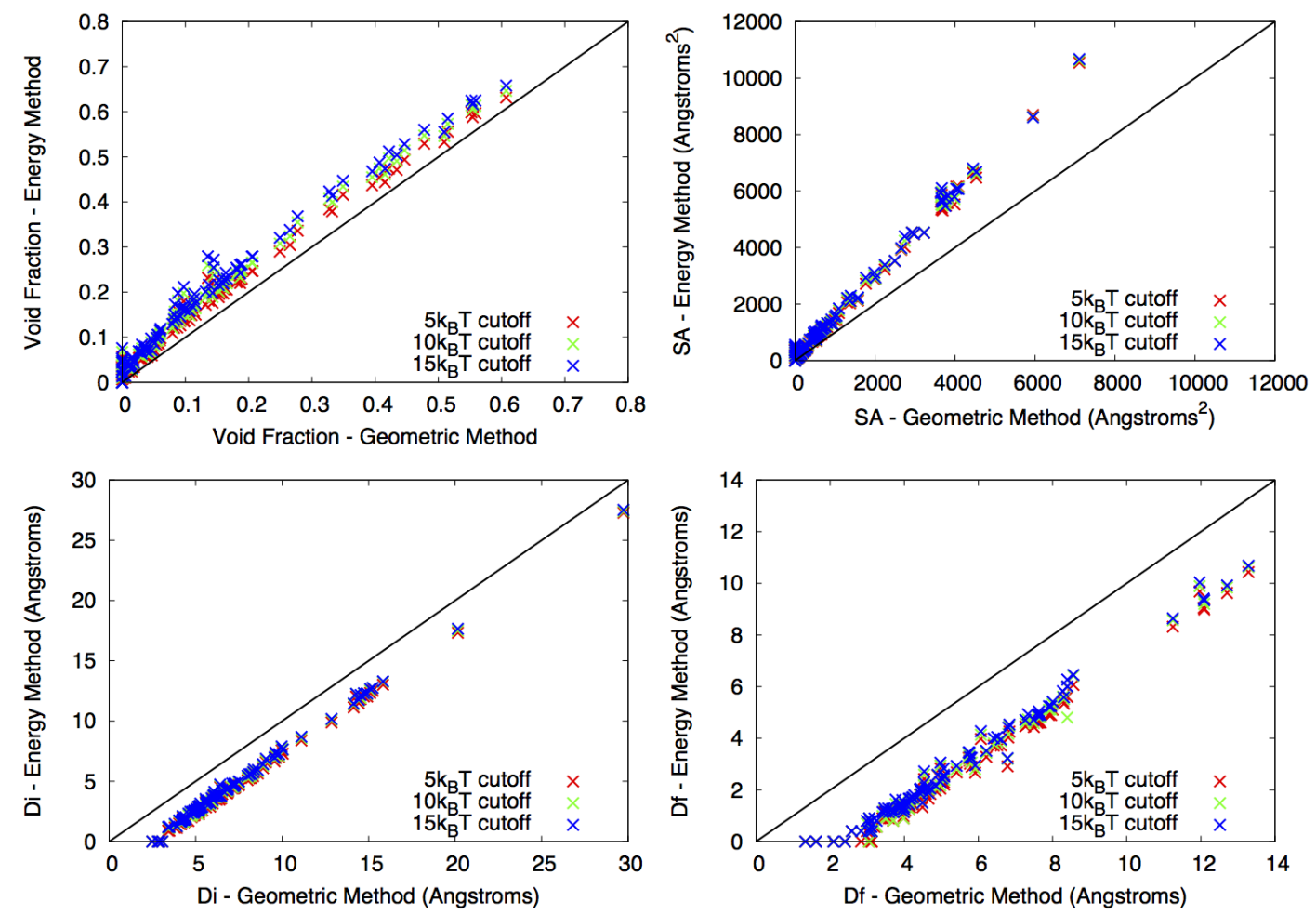

Figure 4. Comparisons between geometric and energy-based methods (red: $5 \mathrm{k}_{\mathrm{B}} \mathrm{T}$ cutoff,

green: $10 \mathrm{k}_{\mathrm{B}} \mathrm{T}$ cutoff, and blue: $15 \mathrm{k}_{\mathrm{B}} \mathrm{T}$ cutoff) for $100 \mathrm{CORE}$ MOF structures using a methane probe. The diagonal black line represents points in which the two methods yield the exact same values.

Next, the effect of the energy (and consequently binary) grid sizes are explored as VF, SA, Di, and DF are re-computed using four different grid sizes $(0.15,0.2,0.25$, and 0.3 Angstrom). In order to expedite analysis, only 20 out of the $100 \mathrm{MOF}$ structures were chosen. Figure 5 shows the results for VF, SA, Di, and Df as the normalized quantities correspond to the ratio between the computational results at $0.2,0.25$, and 0.3 Angstroms against the results at 0.15 Angstroms (which we deem to be the reference value). In general, for grid size of 0.20 Angstroms (' $x$ ' data points in Figure 5), the normalized values are close to one for all 20 structures. Out of the four quantities, Df is most sensitive with 
respect to grid size as depending on the grid, a completely different traversal path can be selected in the binary search. It is worth noting that to retain accurate results, structures
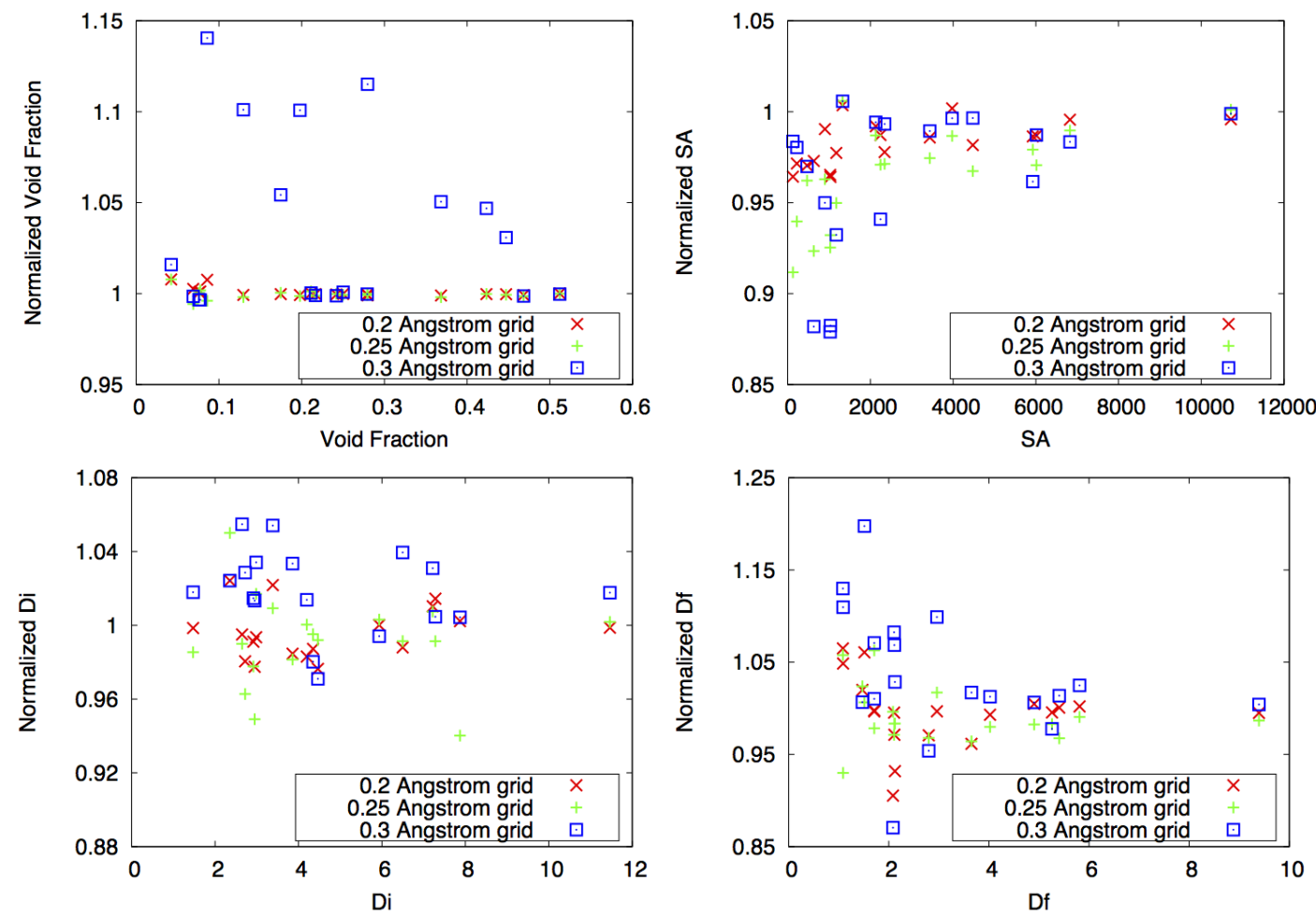

with small Df should be simulated with smaller grid size in general.

Figure 5. Energy-based method results for VF, SA, Di and Df for 20 selected MOF structures at different grid sizes. Normalized quantity refers to the ratio between the results obtained at $0.2,0.25$, or 0.3 Angstroms versus the results obtained at 0.15 Angstroms.

Thus, a normalized value of 1 indicates same values at different grid sizes. VF, SA, Di, and Df values in the horizontal axis refers to the simulation data points at 0.15 Angstroms grid size.

In all of the previous analysis, the methane probe was used as a guest molecule. To demonstrate transferability for a different molecule, the simulations were conducted using a 
helium probe for the same 100 CORE MOF structures. In general, we found there to be a good agreement between the helium results obtained from the energy-based and the geometric-based methods as can be seen from Figure 6. In general, similar trends hold for the helium data points (e.g. larger VF using energy-based method compared to geometricalbased method), which is reasonable as analysis conducted on the methane probe can be applied to the case for helium as well. For Di and Df, the data points have collectively shifted towards the $y=x$ solid line (compared to Figure 4), indicating better agreement between the two methods for the helium probe compared to the case for methane. Given the smaller $\sigma$ value in the Lennard-Jones parameter for helium, the relative distance between the points at the $15 \mathrm{k}_{\mathrm{B}} \mathrm{T}$ cutoff (which is used in the energy-based method) and the center of the framework atoms (which is used in the geometry-based method) become smaller, which accounts for the better agreement for the two methods. 

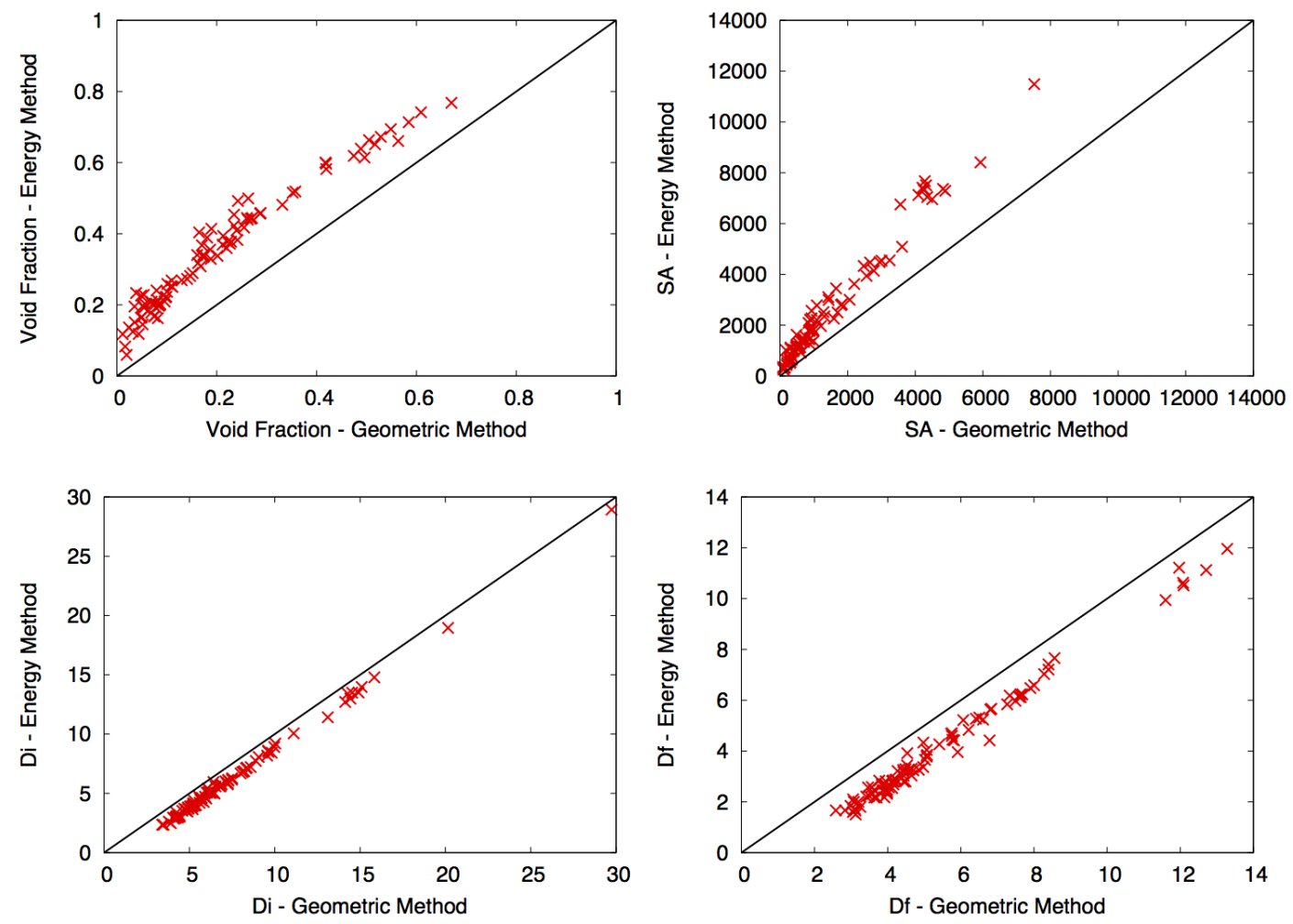

Figure 6. Comparisons between geometric and energy-based methods ( $15 \mathrm{k}_{\mathrm{B}} \mathrm{T}$ cutoff), for 100 CORE MOF structures using a helium probe.

Next, the results between methane and helium probes are compared against one another as a sanity check to ensure that the difference in size (i.e. helium $<$ methane) is properly represented. Figure 7 shows that for VF, Di, and Df, all of the helium values are larger than that for the methane, which is expected given the smaller probe size. For SA, there are few exceptions where methane SA > helium SA. Given that the surface area as computed by the energy-based method relies on the texture of the surface, it is conceivable that the methane probe can result in rough interface between the 0 's and the 1's interface compared to the helium probe, leading to larger SA for the same structure. 

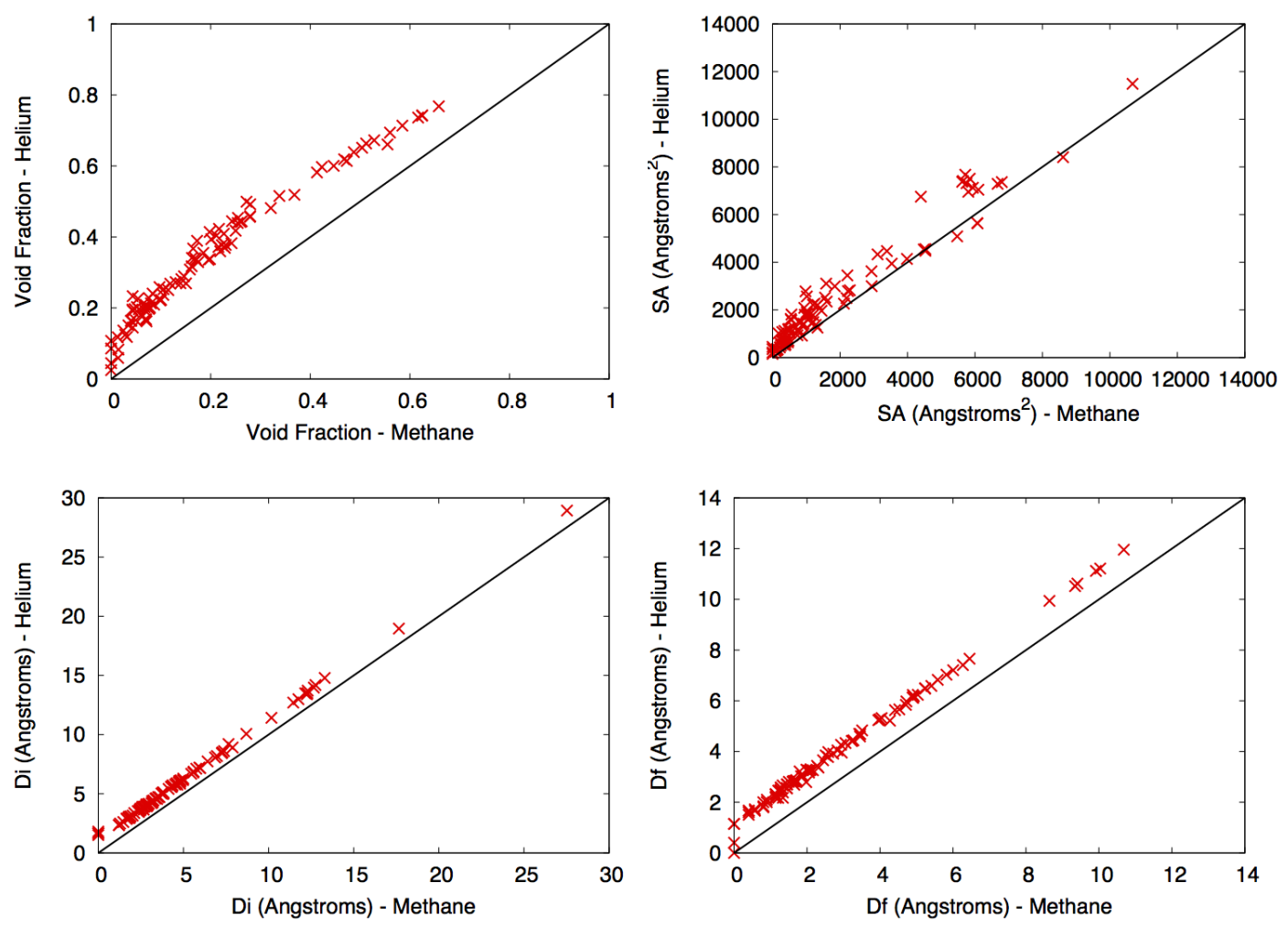

Figure 7. Comparisons between methane and helium probe data for $100 \mathrm{MOF}$ structures using the energy-based $\left(15 \mathrm{k}_{\mathrm{B}} \mathrm{T}\right.$ cutoff).

For large-scale screening applications, it is important to efficiently identify structures that possess the highest void fraction, surface area, and other properties to reduce the number of materials that require further investigations. Given that both the energybased and geometric-based methods are low computationally intensive algorithms, these tools are suitable to use in the preliminary stage of screening. Accordingly, it becomes important that both methods yield the same set of optimal structures even if the exact ordering is not the same. For the test set of $100 \mathrm{MOF}$ structures, the rankings of VF, SA, Di, and Df obtained from energy-based and geometric-based methods are plotted against one another in Figure 8. Again in general, the two methods yield highly correlated data, 
especially amongst the top structures, which is important for screening applications. For low ranked VF materials, the agreement between the two methods become weaker, especially in the case of the helium probe. For these structures that generally possess very low VF values, the ordering does become important to differentiate between completely non-porous and barely porous materials.
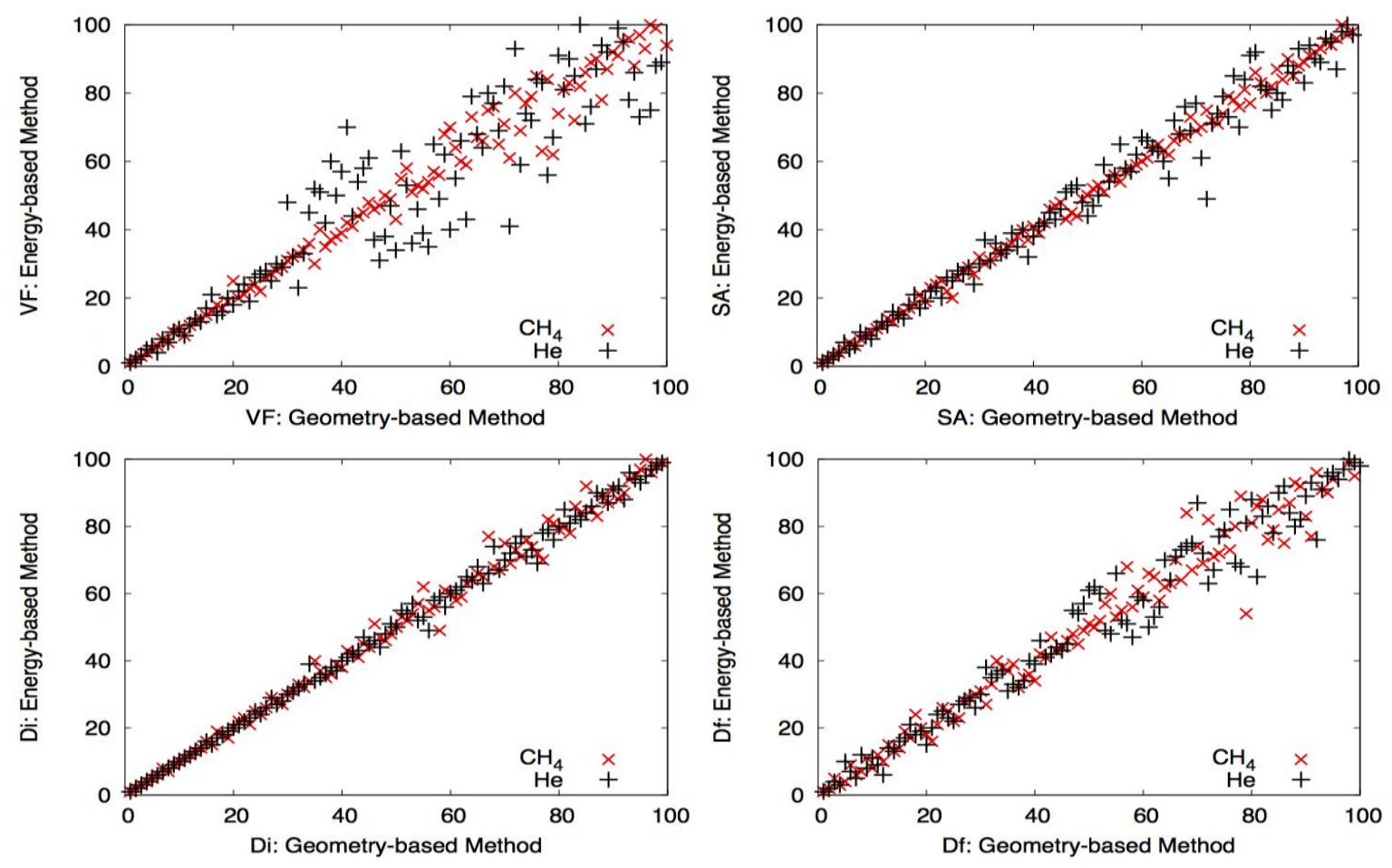

Figure 8. All 100 CORE MOF structures are ranked (from highest to lowest) according to the values obtained from both the energy-based and the geometric-based methods. Their rankings are plotted against one another for both the methane (red) and the helium (black) probes for VF, SA, Di, and Df.

Finally, in order to test transferability for different materials, a test set of 100 IZA zeolite structures were selected and methane/helium probes were used to compute VF, SA, Di, and Df (See Supporting Information Figure S1 and S2). In general, similar trends are 
observed in zeolite structures, indicating that the methodology extends well to different classes of porous material. Ultimately, this transferability depends on the availability of appropriate force-fields.

\section{Conclusion}

We have developed methods to characterize porosity in porous materials using the following descriptors: the void fraction, accessible surface area, largest included sphere, and largest free sphere. Unlike previously introduced methods based on hard sphere approximation (i.e. purely geometry-based), our methods rely on classical force fields to define probe-material interaction energies, and use an energy threshold value to establish the boundary of the material's framework. Therefore, we refer to our methods as energybased methods. In a test set of MOF and zeolite structures, we have observed good agreement between the two methods, indicating the reliability of our energy-based algorithm. One key difference between the two methods is found in structures with small void fractions where energy-based methods (geometric-based methods) indicate presence (absence) of pores. The cutoff values imposed on the energy-based methods can be treated as input parameters that allow users to focus on specific regions of interest (e.g. strong binding sites) and can become informative when it comes to understanding the molecular simulation results obtained from the same set of force field parameters. All of the codes that we have developed are available upon request. 


\section{ACKNOWLEDGMENT}

Dooam Paik and Jihan Kim gratefully acknowledge the financial support from Saudi AramcoKAIST $\mathrm{CO}_{2}$ Management Center. Maciej Haranczyk was supported by the U.S. Department of Energy, Office of Basic Energy Sciences, Division of Chemical Sciences, Geosciences and Biosciences under Award DE-FG02- 12ER16362.

\section{REFERENCES}

[1] N. Rosi, J. Eckert, M. Eddaoudi, D.T. Vodak, J. Kim, M. O’Keeffe, O.M. Yaghi, Science. 300 (2003) 1127-1129.

[2] M. Eddaoudi, J. Kim, N. Rosi, D.T. Vodak, J. Wachter, M. O’Keeffe, O.M. Yaghi, Science. 295 (2002) 469-472.

[3] J. Seo, D. Whang, H. Lee, S. Jun, J. Oh, Y. Jeon, K. Kim, Nature. 404 (2000) 982-986.

[4] D.M. D’Alessandro, B. Smit, J.R. Long, Angew Chem Int Ed Engl. 49 (2010) 60586082.

[5] H. Furukawa, K.E. Cordova, M. O'Keeffe, O.M. Yaghi, Science. 341 (2013) 974-986.

[6] C.E. Wilmer, M. Leaf, C. Lee, O.K. Farha, B.G. Hauser, J.T. Hupp, R.Q. Snurr, Nat Chem. 4 (2011) 83-89.

[7] Database of Zeolite Structures, IZA Structure Commission, 24. Aug. 2015. http://www.iza-structure.org/databases.

[8] Hypothetical-MOFs Database, Northwestern University, 24. Aug. 2015. http://hmofs.northwestern.edu/hc/crystals.php. 
[9] 3D Covalent Organic Framework Materials database, Office of Scientific and Technical Information, 25. Aug. 2015. http://www.osti.gov/dataexplorer/biblio/1155071

[10] T. Düren, Y-S. Bae, R. Snurr, Chem Soc Rev. 38 (2009) 1237-1247.

[11] A. Dzubak, L.C. Lin, K. Kim, J. Swisher, R. Poloni, S. Maximoff, B. Smit, L. Gagliardi, Nat Chem. 4 (2012) 810-816.

[12] K. Sillar, A. Hofman, J. Sauer, J Am Chem Soc. 131 (2009) 4143-4150.

[13] R. Krishna, J.M. Van Baten, E. Garcia-Perez, S. Calero, Chem Phys Lett. 429 (2006) 219-224.

[14] L.C. Lin, A. Berger, R. Martin, J. Kim, J. Swisher, K. Jariwala, C. Rycroft, A. Bhown, M. Deem, M. Haranczyk, B. Smit, Nat Mater. 11 (2012) 633-641.

[15] J. Kim, L.C. Lin, J.A. Swisher, M. Haranczyk, B. Smit, J Am Chem Soc. 134 (2012) 18940-18943.

[16] E. Haldoupis, S. Nair, D.S. Sholl, Phys Chem Chem Phys. 13 (2011) 5053-5060.

[17] C.M. Simon, R. Mercado, S.K. Schnell, B. Smit, M. Haranczyk, Chem Mater. 27 (2015) 4459-4475.

[18] C.M. Simon, J. Kim, D.A. Gomez-Gualdron, J.S. Camp, Y. Chung, R. Martin, B. Smit, Energy Environ Sci. 8 (2015) 1190-1199.

[19] T.F. Willems, C.H. Rycroft, M. Kazi, J.C. Meza, M. Haranczyk, Microporous Mesoporous Mater. 149 (2012) 134-141.

[20] L. Sarkisov, A. Harrison, Mol Simul. 37 (2011) 1248-1257.

[21] E.L. First, C.E. Gounaris, J. Wei, C.A. Floudas, Phys Chem Chem Phys. 13 (2011) 17339-17358. 
[22] T. Düren, F. Millange, G. Ferey, K.S. Walton, R. Snurr, J Phys Chem C. 111 (2007) $15350-15356$.

[23] C.M. Simon, J. Kim, L.C. Lin, R. Martin, M. Haranczyk, B. Smit, Phys Chem Chem Phys. 16 (2014) 5499-5513.

[24] Y. Bao, R.L. Martin, M. Haranczyk, M.W. Deem, Phys Chem Chem Phys. 17 (2015) 11962-11973.

[25] R.L. Martin, M. Haranczyk, Chem Sci. 4 (2013), 1781-1785.

[26] A.K. Rappé, C.J. Casewit, K.S. Colwell, W.A. Goddard $3^{\text {rd }}$, W.M. Skiff, J Am Chem Soc. 114 (1992) 10024-10035.

[27] E. Garcia-Perez, J.B. Parra, C.O. Ania, A. Garcia-Sanchez, J.M. Van Baten, R. Krishna, D. Dubbeldam, S. Calero, Adsorption. 13 (2007) 469-476.

[28] M.G. Martin, J.I. Siepmann, J Phys Chem B. 102 (1998) 2569-2577.

[29] D. Dubbeldam, S. Calero, D. Ellis, R.Q. Snurr, Mol Simul. 42 (2015) 81-101.

[30] M. Pinheiro, R. Martin, C.H. Rycroft, M. Haranczyk, Cryst Eng Comm. 15 (2013) $7531-7538$.

[31] J. Kim, R. Martin, O. Ruebel, M. Haranczyk, B. Smit, J Chem Theory Comput. 8 (2012) 1684-1693.

[32] Y. Chung, J. Camp, M. Haranczyk, B.J. Sikora, W. Bury, V. Krungleviciute, T. Yildirim, O.K. Farha, D.S. Sholl, R.Q. Snurr, Chem Mater. 26 (2014) 6185-6192. 